Related content

\section{Current-induced magnetization reversal in magnetic nanowires}

To cite this article: J.-E. Wegrowe et al 1999 EPL 45626

View the article online for updates and enhancements. edpsciences IOP Institute of Physics
- Magnetization reversal triggered by spin- polarized current in magnetic nanowires
- Magnetic relaxation of nanowires: beyond the Néel-Brown activation process J.-E. Wegrowe, J. P. Meier, B. Doudin et al.
Atomistic observation of magnetization along a hysteresis loop in terbium/iron multilayers withperpendicular magnetic anisotropy inferred from site-selective ${ }^{5 /}$ Fe-Mössbauer spectroscopy
J. Tappert, S. Neumann, R. A. Brand et al.

\section{Recent citations}

- Effective field study of the magnetism and
$\frac{\text { superconductivity in idealised Ising-type }}{\text { X@Y60 endohedral fullerene system }}$
Ersin Kantar
- Magnetic properties of the Ashkin-Teller
$\frac{\text { model on a hexagonal nanotube }}{\text { R.M. Francisco and J.P. Santos }}$
- Magnetic behaviors in a bilayer graphene
nanoisland structure: A Monte Carlo study
Wei Wang et al




\title{
Current-induced magnetization reversal in magnetic nanowires
}

\author{
J.-E. Wegrowe, D. Kelly, Y. Jaccard, Ph. Guittienne and J.-Ph. Ansermet \\ Institut de Physique Experimentale, Ecole Polytechnique Fédérale de Lausanne \\ CH-1015 Lausanne, Switzerland
}

(received 1 October 1998; accepted 23 December 1998)

PACS. 75.40Gb - Dynamic properties (dynamic susceptibility, spin waves, spin diffusion, dynamic scaling, etc.).

PACS. 75.70Pa - Giant magnetoresistance.

PACS. 75.60-d - Domain effects, magnetization curves, and hysteresis.

\begin{abstract}
The effect of pulsed currents on magnetization reversal were studied on single ferromagnetic nanowires of diameter about $80 \mathrm{~nm}$ and $6000 \mathrm{~nm}$ length. The magnetization reversal in these wires occurs with a jump of the magnetization at the switching field $H_{\mathrm{sw}}$, which corresponds to unstable states of the magnetization. A pulsed current of about $10^{7} \mathrm{~A} / \mathrm{cm}^{2}$ was injected at different values of the applied field close to $H_{\mathrm{sw}}$. The injected current triggered the magnetization reversal at a value of the applied field distant from the switching field by as much as $20 \%$. This effect of current-induced magnetization reversal is interpreted in terms of the action of the spin-polarized conduction electrons on the magnetization.
\end{abstract}

The first discovered spin-dependent scattering process, the anisotropic magnetoresistance (AMR), arises from the anisotropy of the ferromagnetic scattering centers [1]. The recent discoveries of Giant Magnetoresistance (GMR) [2], Domain Wall Scattering (DWS) [3], and Tunnel Magnetoresistance [4] (TMR) have shown the variety of spin-dependent scattering processes which can occur in nanostructured ferromagnetic materials. GMR or DWS can be thought of as the response of the current to a gradient of the magnetization. In this letter, we give experimental evidence to the reverse effect: the response of the magnetization to an electric current.

The force applied by polarized conduction electron spins on ferromagnetic moments has been predicted theoretically by different approaches [5,6]. Experimental investigation has been performed by Hung and Berger [7] on a macroscopic sample with pinned domain walls, where an effect of the current has been evidenced. However, due to the macroscopic scale of the system, stable magnetization states separated by unstable states (or jump of the magnetization) could not be observed. Recently, Tsoi et al. have evidenced the action of a high current at a point contact on spin waves generation [8].

The aim of this paper is to show the action of the current on the magnetization of single nanowires of about $10^{-11} \mathrm{emu}\left(10^{-14} \mathrm{~A} \mathrm{~m}^{2}\right)$ and how it can be interpreted in terms of conduction electron spin polarization.

(C) EDP Sciences 
Samples. - The experiments were performed on various single $\mathrm{Ni}$ nanowires at room temperature. The wires were obtained by electrodeposition in porous membranes used as templates. The average pore diameter in the membrane was about $80 \pm 10 \mathrm{~nm}$. The length of the wire was $6000 \mathrm{~nm}$. The description of the deposition process [9], the study of the polycrystalline structure, magnetic configurations and effects of the distribution inside the membrane have been characterized [10]. The magnetization reversal of single nanowires has been studied using micro-SQUID technique [11] and by measuring the AMR with microcontacts on the membrane $[12,13]$. In a single $\mathrm{Ni}$ nanowire, the magnetization reversal is reversible up to a given value, the so-called switching field $H_{\mathrm{sw}}$, where the magnetization jumps irreversibly to a reversed direction. This jump corresponds to unstable states of the magnetization. In the case of $\mathrm{Ni}$ wires of small diameter, the magnetization states can be described quantitatively, as a function of the amplitude and the direction of the applied field in terms of the curling mode of magnetization reversal $[11,13]$. In the case of Co nanowires [13], in the case of larger Ni wires [11], or in the presence of magnetic or topological defects in the membrane $[10,14]$, the magnetization reversal occurs with more complicated spin configurations, like vortex or domain walls [15]. In the samples used here, the contacts are performed with a thin electrodeposited Ni layer. This layer plays the role of a magnetic defect and may induce a vortex or domain wall in the wire.

Experiments. - Figure 1(a) shows the magnetoresistive hysteresis loop of a single $\mathrm{Ni}$ nanowire, measured at small angle $\theta$ of the applied field with respect to the wire axes. The magnetoresistive curve is measured with $0.5 \mu \mathrm{A}$ current $\left(10^{4} \mathrm{~A} / \mathrm{cm}^{2}\right)$ with a lock-in detection. The consecutive magnetization states, measured with a sweeping rate of $1 \mathrm{Oe} / \mathrm{s}$, could be described qualitatively in the following way (fig. 1(b)). At large negative fields the magnetization is saturated in the down direction (I). At about $-0.1 \mathrm{kOe}$, the transverse component of the magnetization appears (II). The minimum of the magnetoresistance at about $+0.2 \mathrm{kOe}$ corresponds to the largest contribution of transverse magnetization. At about $+0.3 \mathrm{kOe}$, some unstable states of the magnetization is reached (III) before the jump of the magnetization occurs. Then, the magnetization is saturated in the up direction (IV). The two states before and after the jump can be reproduced in many magnetoresistive hysteresis loops (fig. 1(c)). The switching field $H_{\mathrm{sw}}$ is well defined at $0.43 \mathrm{kOe}$. Individual jumps are measured with the accuracy of the field step, i.e. about 5 Oe. The same magnetoresistive hysteresis loops are performed with a $200 \mathrm{~ns}$ pulsed current of amplitude $0.15 \mathrm{~mA}$ (about $10^{7} \mathrm{~A} / \mathrm{cm}^{2}$ ) injected at an applied field of $0.33 \mathrm{kOe}$. The injected current provokes the magnetization reversal at this field (fig. 1(d)). The magnetization reversal occurs hence at a new switching field: $H_{\mathrm{sw}}^{i}=0.33 \mathrm{kOe}$. A current injected at position between $H_{\mathrm{sw}}^{i}$ and $H_{\mathrm{sw}}$ provokes also the jump of the magnetization. The maximum switching field variation $\Delta H_{\max }=\left|H_{\mathrm{sw}}^{i}-H_{\mathrm{sw}}\right|$ produced by the pulsed current is defined by the minimum field $H_{\mathrm{sw}}^{i}$ at which the current induces the jump.

The parameter $\Delta H_{\max }$ was measured on another sample vs. the angle of the applied field (see fig. 2). The magnetoresistive hysteresis loops measured at $\theta=70^{\circ}$ and $\theta=20^{\circ}$ can be compared. In order to take into account the fluctuations due to defects and other statistical events, histograms of the $H_{\mathrm{sw}}$ are obtained by repeating the measurement about 80 time per switching field (fig. 2(c) and fig. 2(d)). For a given angle the histogram without pulsed current is plotted together with the histogram with pulsed current taken at the maximum distance $\Delta H_{\max }$. The two distributions are separated by 100 to 200 Oe.

Other effects of pulsed current. - Spurious effects of the current must be ruled out.

(a) The magnetic field induced by the current $I$ is poloidal and its value at a distance $a$ of the axes of the wire of radius $r$, is given by $B=\frac{2 I}{r^{2}} a$. The maximal field, for $r=a=30 \mathrm{~nm}$ 

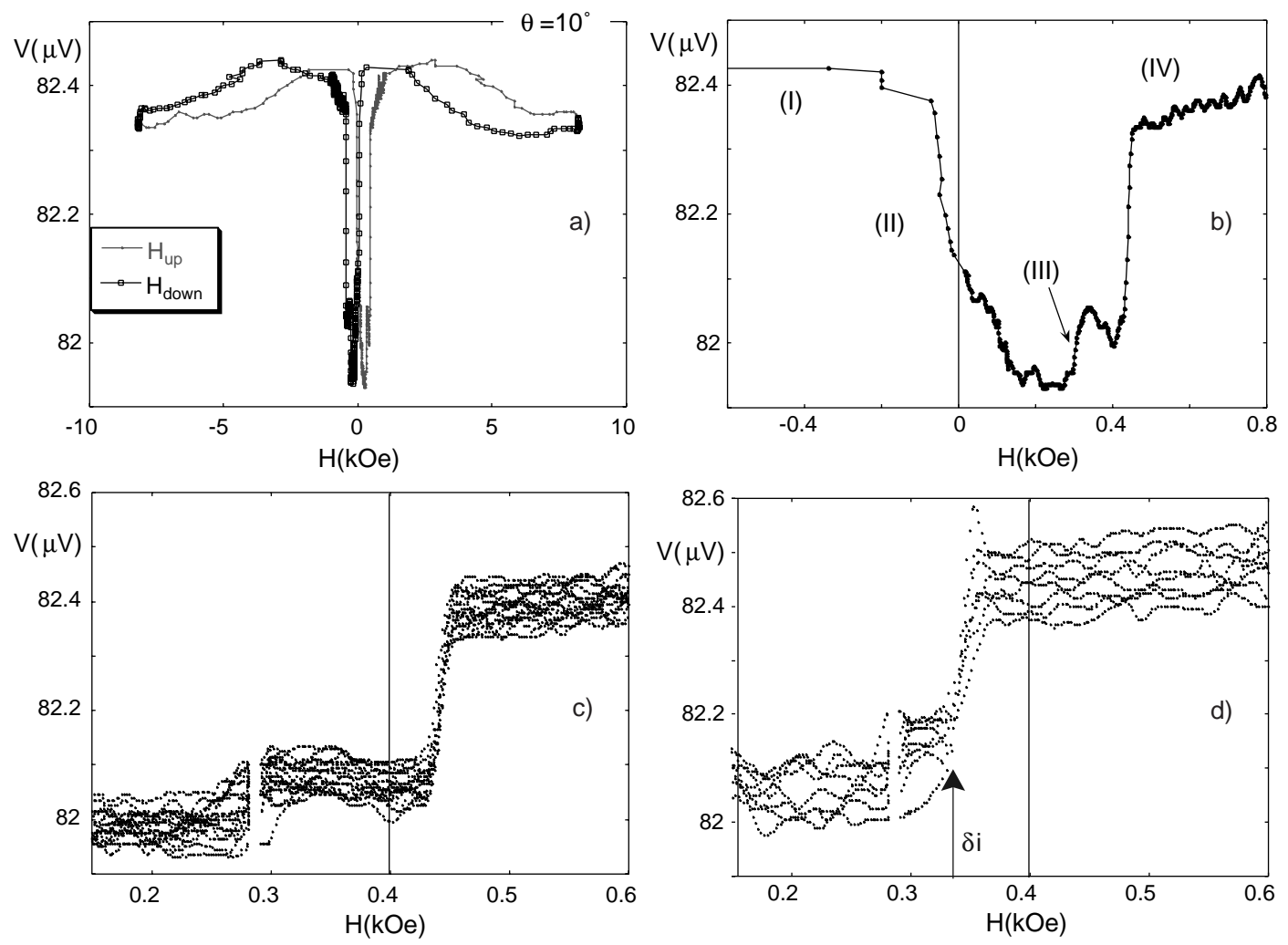

Fig. 1. - (a) Magnetoresistive hysteresis loop measured at $\theta=10^{\circ}$ with a reading current of $0.5 \mu \mathrm{A}$. (b) Zoom of (a). The magnetization states corresponding the area (I), (II), (III) and (IV) of the magnetoresistance are described in the text. (c) Zoom without pulsed current. The switching field is $H_{\mathrm{sw}}=0.43 \mathrm{kOe}$. (d) Same hysteresis with pulsed current at $H=0.33 \mathrm{kOe}$. The switching field is now $H_{\mathrm{sw}}^{i}=0.33 \mathrm{kOe}$.

gives $B_{\max }=10$ Oe for $I=0.15 \mathrm{~mA}$. This value is about one order of magnitude below that of $\Delta H_{\max }$.

(b) The maximum heating due to the pulsed current can be estimated in the worst case where the Joule power is injected in the center of the wire. The heat can be dissipated at both ends of the wire (at a distance $L=3 \mu \mathrm{m}$ from the spot, through a section $S=\pi \cdot 10^{-15} \mathrm{~m}^{2}$ ), and in the membrane (say at a distance $\delta=500 \mathrm{~nm}$ from the wire, through a surface $\left.A=10^{-12} \mathrm{~m}^{2}\right)$. The thermal conductivity in the wire and in the membrane are taken to be, $\lambda_{\mathrm{Ni}}=90.9\left(\mathrm{~W} \cdot \mathrm{m}^{-1} \cdot \mathrm{K}^{-1}\right)$ and $\lambda_{\mathrm{mb}}=0.36\left(\mathrm{~W} \cdot \mathrm{m}^{-1} \cdot \mathrm{K}^{-1}\right)$. We find that the temperature rise is smaller than $5 \mathrm{~K}$. The temperature dependence of the switching field can be estimated by using measurements of the coercive field. Thus a variation $\Delta H_{\mathrm{c}}=\left(H_{\mathrm{c}}(300)-H_{\mathrm{c}}(295 \mathrm{~K})\right)<20 \mathrm{Oe}$ is expected for a $5 \mathrm{~K}$ variation [10]. $\Delta H_{\mathrm{c}}$ is about 5 times smaller than $\Delta H_{\max }$.

Discussion: the action of the electric current on the magnetization reversal. - We use in the following the description of the effect of the polarized conduction electron spins proposed recently by Bazaliy et al. [6]. These authors show that the interaction between the conduction electron spins and the magnetic moment can be reduced to an additive term in the effective field $\mathbf{H}_{\text {eff }}=\frac{\partial V}{\partial \mathbf{M}}+\frac{\partial V^{\mathrm{I}}}{\partial \mathbf{M}}$ acting on the magnetic moment in the Landau-Lifshitz equation. The energy 

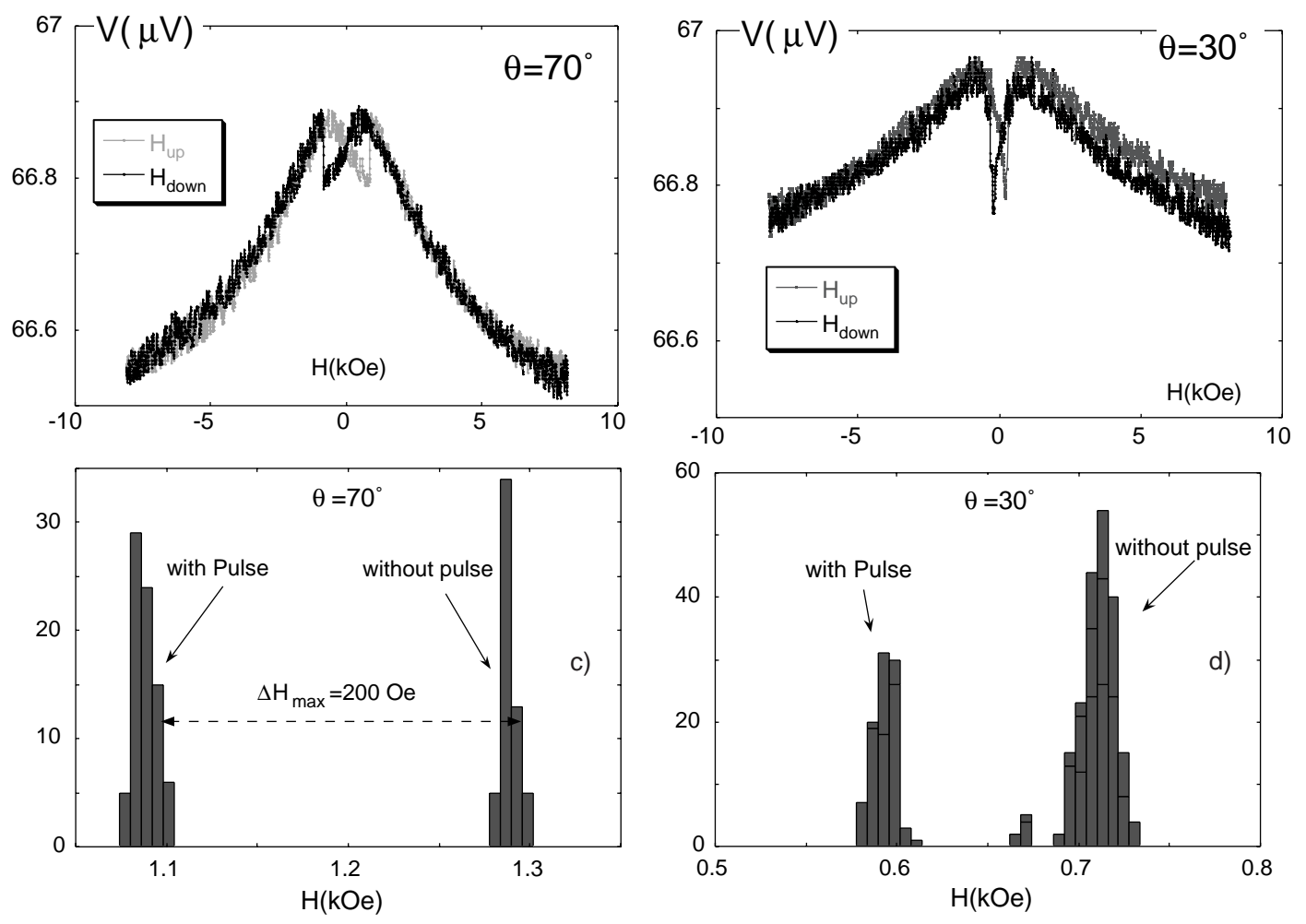

Fig. 2. - (a) Magnetoresistive hysteresis loop measured at $\theta=70^{\circ}$ and (b) at $\theta=30^{\circ}$. (c) Histogram of the switching field with and without pulsed current at $\theta=70^{\circ}$ and (d) at current at $\theta=30^{\circ}$.

$V$ contains the Zeeman energy $\mathbf{M} \cdot \mathbf{H}_{\mathrm{ex}}$ due to the external field $\mathbf{H}_{\mathrm{ex}}$, the anisotropy energy, the dipolar energy, and the exchange energy. The energy $V^{\mathrm{I}}$ is due to the interaction between polarized conduction electron spins and the ferromagnetic moments. When the conduction electrons experience a change in the magnetization over a distance smaller than the spin diffusion length $\lambda_{\text {sf }}$, then the polarized conduction electron spins relax $[2,3,5]$. At this point, the current produces a rotation $\Delta \varphi$ of the magnetization from it position $\varphi$ in the absence of current $[6]$.

In the framework of studies of the magnetization reversal in nanoparticles, the magnetization states are described by the hysteresis loop $\mathbf{M}\left(\mathbf{H}_{\mathrm{ex}}\right)$, where the magnetic moment $\mathbf{M}\left(\mathbf{H}_{\mathrm{ex}}\right)$ is at the angle $\varphi$ taken with respect to the wire axis. The magnetic moment follows reversibly the external field up to the switching field $H_{\text {sw }}$ where a jump of the magnetization occurs $[11-13,16]$. After the jump the hysteresis is completed by a second reversible part. The jump occurs at a critical angle $\varphi_{\mathrm{c}}$ [17] of the magnetization. If the current is injected at fields $H_{\mathrm{sw}}^{i}$ corresponding to the magnetic state defined by the angle $\varphi=\varphi_{\mathrm{c}}-\Delta \varphi$, then a jump occurs. The jump would also occur if the current is injected at any field between $H_{\mathrm{sw}}^{i}$ and $H_{\mathrm{sw}}$. The maximum distance $\Delta H_{\mathrm{max}}=H_{\mathrm{sw}}^{i}-H_{\mathrm{sw}}$ constitutes a measure of the angular variation $\Delta \varphi$ produced by the current.

In order to further analyze our data (fig. 3), we need to link the switching field to the angle of the magnetization $\varphi_{\mathrm{c}}$. This question has been solved in some simple cases only, namely in the case of some monodomain magnetization reversal processes [17]. The description used 


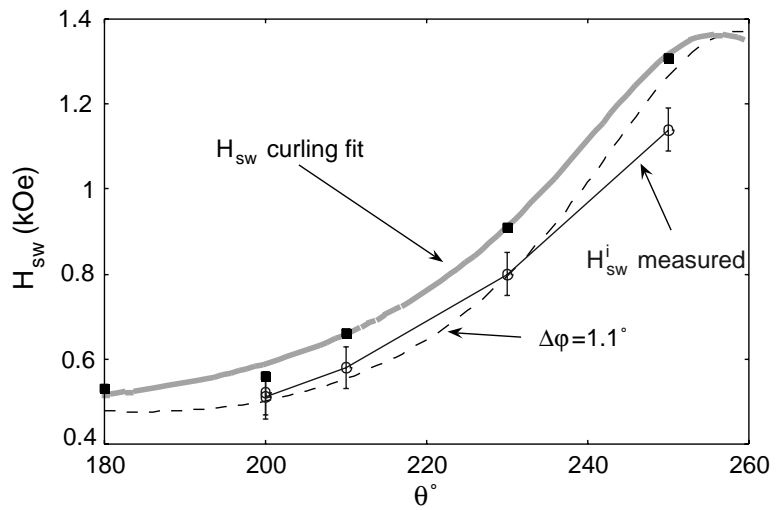

Fig. 3. - Angular dependence $H_{\mathrm{sw}}(\theta)$ of the switching field without pulse and angular dependence $H_{\mathrm{sw}}^{i}(\theta)$ of the minimum switching field with pulsed current. Gray line: $H_{\mathrm{sw}}(\theta)$ fitted with eq. (1). Dashed line: $H_{\mathrm{sw}}^{i}(\theta)$ fitted with eq. (6) assuming $\Delta \varphi=1.1^{\circ}$.

below is valid for the curling reversal mode [13]. In our case, the magnetization reversal may not be uniform before the instability and more complicated spin configurations may occur. Hence, the application of the above model could not be rigorous, but it is able to give an idea of the angular variation $\Delta \varphi$ produced by the current (where the angle $\varphi$ would then be the angle defined by the magnetization inside a domain). It has indeed been shown that the mechanism of magnetization reversal by nucleation of a part only of the sample is very close to that of "true nucleation", i.e. by nucleation from fully saturated state $[11,13,18,19]$.

For the sake of simplicity, we describe the switching field by [17]

$$
h_{\mathrm{sw}}(\theta)=\frac{a(a+1)}{\sqrt{a^{2}+(2 a+1) \cos ^{2}(\theta)}},
$$

where $h_{\mathrm{sw}}$ is the reduced switching field: $h_{\mathrm{sw}}=H(\theta) / H_{a}, H_{a}$ the anisotropy field (about $1.85 \mathrm{kOe}$ ). The fitting parameter is $a=-k\left(R_{0} / r\right)^{2}$, where $k$ is a geometrical parameter and $R_{0}$ is the exchange length [20].

Within the model, the relation between the angle of the applied field $\theta$ and the angle of the magnetization $\varphi$ is

$$
\tan (\theta)=\frac{a+1}{a} \tan (\varphi)
$$

For small variations, we have

$$
\Delta \theta \approx \frac{1+2 a+2 a^{2}+(1+2 a) \cos (2 \theta)}{2 a(1+a)} \Delta \varphi .
$$

We assume that the action of the current is to cause an angular variation $\Delta \varphi$ of the orientation of the ferromagnetic moment. If this variation is sufficient to trigger the magnetization switching (i.e. if $\varphi+\Delta \varphi \geq \varphi_{\mathrm{c}}$ ) the switching field $h_{\mathrm{sw}}$ is shifted to the switching field $h_{\mathrm{sw}}^{i}$. To $\Delta \varphi$ corresponds a $\Delta h$ variation given by

$$
\Delta h(\theta)=\frac{\partial h_{\mathrm{sw}}}{\partial \theta} \cdot \Delta \theta .
$$


Using (1) and (3) yields

$$
\Delta h_{\mathrm{sw}}(\theta)=\frac{(1+2 a) \cos (\theta) \sin (\theta)\left(1+2 a+2 a^{2}+(1+2 a) \cos (2 \theta)\right)}{2\left(a^{2}+(1+2 a) \cos (\theta)^{2}\right)^{3 / 2}} \Delta \varphi
$$

with

$$
h_{\mathrm{sw}}^{i}(\theta)=h_{\mathrm{sw}}(\theta)+\Delta h_{\mathrm{sw}}(\theta) .
$$

Mean values of $H_{\mathrm{sw}}(\theta)$ and $H_{\mathrm{sw}}^{i}(\theta)$ deduced from the histograms are plotted vs. the angle of the applied field (fig. 3). Equation (1) accounts for $H_{\mathrm{sw}}(\theta)$ with $a \approx-0.28$. This value corresponds to an activation radius of $r=30 \pm 10 \mathrm{~nm}$, which coincides approximately with the wire radius. The hypothesis of a set $\Delta \varphi$ for a set pulsed current accounts reasonably well for our data (fig. 3) [21]. The application of eqs. (5) and (6) on the measured data $H_{\mathrm{sw}}^{i}(\theta)$ leads to the dashed line in fig. 3 , taking $\Delta \varphi \approx 1.1^{\circ}$. In ref. [6] a theoretical estimate of the angle $\Delta \varphi$ is given as a function of the current $j$ (the angle $\Delta \varphi$ is noted $\theta_{0}$ in [6] and is deduced by a continuity equation through the interface). The angle variation $\Delta \varphi=1.1^{\circ}$ corresponds

to a current of about $j=0.1 j_{0}$, where $j_{0}=\frac{e}{\hbar} \sqrt{K J M^{2}}$ is the characteristic current. The constant $K$ is the anisotropy, $J$ the exchange constant and $M$ is the magnetic moment at saturation [6]. We deduce a current density of $j=4 \cdot 10^{6} \mathrm{~A} / \mathrm{cm}^{2}$, where we used the shape anisotropy $K_{a}=5 \cdot 10^{5}\left(\mathrm{erg} / \mathrm{cm}^{3}\right)$ of our wire. The agreement between this estimate and the effective injected pulsed current of about $10^{7} \mathrm{~A} / \mathrm{cm}^{2}$ is reasonable, considering the rough approximations we used.

Conclusion. - The current density which produced the magnetization reversal is of the right order of magnitude according to the available models of magnetic excitation by currents $[12,8,11]$. The effects of heating and induced fields are not sufficient alone to be responsible for the magnetization reversal. Our measurements give hence strong evidence that the magnetization reversal is driven by the action of the spin-polarized current. Our observations also demonstrate the feasibility of a simple writing process for magnetoresistive memories based on assembly of nanowires [22].

\section{REFERENCES}

[1] McGuire T. R. and Potter R. I., IEEE Trans. Magn., MAG-11 (1975) 1018; Rijks Th. G. S. M., Coehoorn R., De Jong M. J. M. and De Jonge W. J. M., Phys. Rev. B, 51 (1995) 283.

[2] Baibich M. N., Broto J. M., Fert A., Nguyen Van Dau F. and Petroff F., Phys. Rev. Lett., 61 (1988) 2472.

[3] Gregg J. F., Allen W., Ounadjela K., Viret M., Hehn M., Thompson S. M. and Coey J. M. D., Phys. Rev. Lett., 77 (1996) 1580; Levy P. and Shufeng Zhang, Phys. Rev. Lett., 79 (1997) 5111.

[4] Merservey R. and Tedrow P. M., Phys. Rep., 238 (1994) 173.

[5] Cabrera G. C. and Falikov L. M., Phys. Status solidi B, 61 (1974) 539; Berger L., J. Appl. Phys., 55 (1984) 1954; Johnson M. and Silbee R. H., Phys. Rev. B, 37 (1987) 5312; Slonczewski C., J. Magn. \& Magn. Mater., 159 (1996) L1.

[6] Bazaliy Ya. B., Jones B. A. and Shou-Chen Zhang, Phys. Rev. B, 57(1998) R3213.

[7] Hung C.-Y. and Berger L., J. Appl. Phys., 63 (1998) 4276.

[8] Tsoi M., Jansen A. G. M., Bass J., Chiang W.-C., Seck M., Tsoi V. and Wyder P., Phys. Rev. Lett., 80 (1998) 4281.

[9] Martin C. R., Science, 266 (1994) 1961.

[10] Meier J., Doudin B. and Ansermet J.-Ph., J. App. Phys., 79 (1996) 6010; Beeli C., Doudin B., Ansermet J.-Ph. and Stadelmann P., J. Magn. E Magn. Mater., 164 (1996) 77. 
[11] Wernsdordfer W., Hasselbach K., Benoit A., Barbara B., Doudin B., Meier J. and Ansermet J.-Ph., Phys. Rev. Lett., 77 (1996) 1873; Wernsdordfer W., Hasselbach K., Benoit A., Barbara B., Doudin B., Meier J. and Ansermet J.-Ph., Phys. Rev. B, 55 (1997) 11552.

[12] Wegrowe J.-E., Gilbert S. E., Kelly D., Doudin B. and Ansermet J.-Ph., IEeE Trans. Mag., 34 (1998) 903.

[13] Wegrowe J.-E., Kelly D., Franck A., Gilbert S. E. and Ansermet J.-Ph., submitted to Phys. Rev. Lett.

[14] Wegrowe J.-E., Meier J. P., Doudin B., Ansermet J.-Ph., Wernsdorfer W., Barbara B., Coffey W. T., Kalmykov Y. P. and DéJardin J.-L., Europhys. Lett., 38 (1997) 329.

[15] A visualization of the magnetic configurations by numerical simulations can be found in FERRÉ R., Ounadjela K., George J. M., Piraux L. and Dubois S., Phys. Rev. B, 56 (1997) 14066 or Shui S. T. and De-Cheng Tian, J. Appl. Phys., 78 (1997) 6128.

[16] Adeyeye A. O., Bland J. A. C., Daboo C. and Hsko D. G., Phys. Rev. B, 56 (1998) 3265; Hong K. and Giordano N., J. Phys. Condens. Matter, 10 (1998) L401.

[17] Aharoni, Introduction to the Theory of Ferromagnetism (Clarendon Press, Oxford) 1996, and reference therein.

[18] Fruchart O., Wernsdorfer W., Nozières J.-P., Givord D., Rousseaux F., Mailly D., Decanini D. and Carcenac F., Proceedings of MML98 Conference (Vancouver), to be published in J. Magn. 8 S Magn. Mater.; Wegrowe J.-E., Fruchart O., Nozières J.-P., Ansermet J.-Ph., Givord D., Rousseaux F., Decanini D. and Carcenac F., to be published in $J$. Appl. Phys.

[19] Kronmueller H., Durst K.-D. and MartineK G., J. Magn. 6 Magn. Mater., 69 (1987) 149D; Givord D., Tenaud P. and Viadieu T., J. Magn. E Magn. Mater., 72 (1988) 247; Cebollada, Rossignol M. F. and Givord D., Phys. Rev. B, 52 (1995) 13511.

[20] Aharoni, J. Appl. Phys., 30 (1959) 70S.

[21] This hypothesis is justified by the fact that both the nature of the domain wall $\left(180^{\circ}\right.$ between two domains) and the domain wall thickness (limit of stability) are roughly constant whatever the orientation of the domain with respect to the wire axis. Furthermore, the discrepancy observed at large angle can be understood by the effect of the geometry of the current lines with respect to the domain wall. Namely the difference between CPW (current perpendicular to the wall) and CIW (current parallel to the wall) effects [3].

[22] Patent filed. 